\section{Rethink the classical view of cerebrospinal fluid production}

\author{
Margaux Roques (D), Amaury De Barros and Fabrice Bonneville
}

In their brilliant review, Joanna Wardlaw and colleagues describe those well-known but poorly understood CNS features, the perivascular spaces (Wardlaw, J. M. et al. Perivascular spaces in the brain: anatomy, physiology, and pathology. Nat. Rev. Neurol. 16, 137-153 $\left.(2020)^{1}\right)$. Questions persist on the role of these spaces in interstitial fluid-cerebrospinal fluid (ISF-CSF) metabolite clearance, such as drainage pathways, the role of aquaporin 4 in water transport, and the effects of sleep on their function. In their discussion, the authors accurately indicate that the occurrence of CSF drainage via arachnoid granulations in humans has been questioned. We believe that their statement that CSF is "produced continuously in the choroid plexus" should be examined too.

The traditional 'bulk flow' theory of active production of CSF in the choroid plexus and its passive absorption via arachnoid granulations - historically presented as the 'third circulation' - mostly originates from a single experiment by Dandy published in 1919 that has not been reproduced since. He performed a unilateral choroid plexectomy and obstruction of the foramen of Monro on one dog, which led to the collapse of the plexectomized ventricle and dilation of the ventricle containing a choroid plexus ${ }^{2}$. By contrast, in 1969, Milhorat et al. ${ }^{3}$ obstructed the ventricular system of 76 monkeys, which caused hydrocephalus independently of choroid plexectomy, suggesting a role for extra-choroidal sites in CSF production.

The function of the choroid plexus has frequently been investigated in in vitro and ex vivo experiments, the conditions of which could differ from physiological conditions. However, MRI can be used to explore CSF dynamics in vivo. Use of dynamic oxygenenhanced MRI has demonstrated that inhaled oxygen predominantly diffuses into the sulcal CSF rather than the ventricular $\mathrm{CSF}^{4}$. This observation could be explained by the abundance of pial arterioles relative to intraventricular vessels and the direct crossing of intra-arterial molecules through the blood-CSF barrier to form CSF. In addition, use of several imaging techniques in which CSF motion is directly visualized has demonstrated no motion around the choroid plexus, which contradicts the notion that choroid plexus arterial pulsation is the driving force for CSF pulsatile motion 5 . These in vivo findings support a new model, in which CSF is produced by water filtration across capillary walls throughout the CNS and is subjected to a combination of multidirectional motions, where hydrodynamic and osmotic changes play a crucial role 6 .

Another line of evidence that suggests that the choroid plexus is not the only site of CSF production is the failure of plexectomy to treat paediatric hydrocephalus. This approach, which was used for decades, has largely been replaced by shunt or endoscopic ventriculostomy owing to its lack of efficacy in all but exceptional cases of CP hyperplasia ${ }^{7}$. In addition, the combination of choroid plexus cauterization with ventriculostomy does not improve outcomes for children with post-infectious hydrocephalus ${ }^{8}$. Inflammation has recently been proved to play a prominent role in acquired hydrocephalus, but further experiments are required for greater clarity ${ }^{9}$.

In summary, the oversimplified bulk flow theory is commonly presented as fact in textbooks and medical schools but should be presented as one of several models, along with its limitations and flaws. Examination of the entire ISF-CSF pathway from production to clearance is essential for improving treatment of conditions that involve altered CSF dynamics, such as obstructive hydrocephalus, normal pressure hydrocephalus and even intracranial hypotension, by taking into consideration not only the symptoms but, above all, the responsible mechanisms.

There is a reply to this letter by Wardlaw et al. Nat. Rev. Neurol. https://doi.org/10.1038/ s41582-021-00539-z (2021).

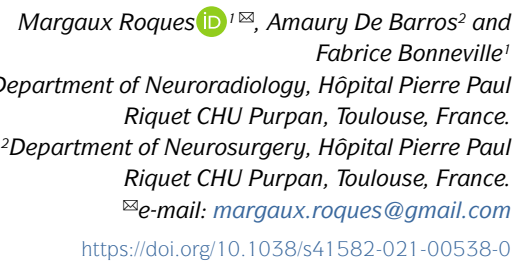

1. Wardlaw, J. M. et al. Perivascular spaces in the brain anatomy, physiology and pathology. Nat. Rev. Neurol. 16, 137-153 (2020)

2. Dandy, W. E. Experimental hydrocephalus. Ann. Surg 70, 129-142 (1919)

3. Milhorat, T. H., Hammock, M. K., Fenstermacher, J. D. Rall, D. P. \& Levin, V. A. Cerebrospinal fluid production by the choroid plexus and brain. Science 173 330-332 (1971)

4. Mehemed, T. M. et al. Dynamic oxygen-enhanced MRI of cerebrospinal fluid. PLOS ONE 9, e 100723 (2014).

5. Matsumae, M. et al. Changing the currently held concept of cerebrospinal fluid dynamics based on shared findings of cerebrospinal fluid motion in the cranial cavity using various types of magnetic resonance imaging techniques. Neurol. Med. Chir. 59, 133-146 (2019).

6. Orešković, D., Radoš, M. \& Klarica, M. New concepts of cerebrospinal fluid physiology and development of hydrocephalus. Pediatr. Neurosurg. 52, 417-425 (2017).

7. Limbrick, D. D., Baird, L. C., Klimo, P., Riva-Cambrin, J. \& Flannery, A. M. Pediatric hydrocephalus: systematic literature review and evidence-based guidelines. Part 4: Cerebrospinal fluid shunt or endoscopic third ventriculostomy for the treatment of hydrocephalus in children. J. Neurosurg. Pediatr. 14 30-34 (2014).

8. Kulkarni, A. V. et al. Endoscopic treatment versus shunting for infant hydrocephalus in Uganda. N. Engl. J. Med. 377, 2456-2464 (2017).

9. Karimy, J K et al Inflammation in acquired hydrocephalus: pathogenic mechanisms and therapeutic targets. Nat. Rev. Neurol. 16, 285-296 (2020).

\title{
Reply to: Rethink the classical view of cerebrospinal fluid production
}

\section{Joanna M. Wardlaw (D), Helene Benveniste, Maiken Nedergaard(D), Berislav V. Zlokovic, Serge Charpak, Kenneth J. Smith (1) and Sandra E. Black(D)}

We thank Roques and colleagues for their comments on our Review (Wardlaw, J. M. et al. Perivascular spaces in the brain: anatomy, physiology, and pathology. Nat. Rev. Neurol. 16, 137-153 $\left.(2020)^{1}\right)$, in which they correctly point out some textbook dogma that needs to be re-evaluated (Roques, M., De Barros, A. \& Bonneville, F. Rethink the classical view of cerebrospinal fluid production. Nat. Rev. Neurol. https://doi.org/10.1038/ s41582-021-00538-0 (2021 $\left.)^{2}\right)$. We agree that the questions of how much cerebrospinal fluid (CSF) is produced and from which structures remain unanswered. In our Review, we noted that there are several potential sources of CSF, including the choroid plexus, but information about the relative contributions of each source is unfortunately limited.

In our Review ${ }^{1}$, we focused on perivascular spaces as seen in vivo on human brain 
imaging, how these relate to perivascular spaces as described histologically, and their role in brain fluid management. Perivascular spaces themselves have been a controversial topic since the 1800s, and clarification of their role, along with a full understanding of brain fluid management, would be of great value.

Some evidence supports the existence of several sources of interstitial fluid and CSF and several sites of their drainage from the cranial cavity, including a return to the venous circulation via arachnoid granulations and intradural channels ${ }^{3}$, and drainage via meningeal lymphatics ${ }^{1,4}$, including via skull base lymphatics to nasal lymphatics ${ }^{5}$. However, the major remaining questions concern how much fluid is produced and removed by each route, and whether the function of these pathways differs by age, sex, brain and physical activity (including sleep and wakefulness) and disease states.

The choroid plexus is generally agreed to be a key site of CSF production ${ }^{4}$, although the relative contributions of choroid plexus in the lateral ventricle versus that in the fourth ventricle are uncertain and may differ between species. There are also longstanding arguments that interstitial fluid contributes to the CSF and ultimately to lymphatic drainage $^{4,6,7}$. Water is a byproduct of metabolic reactions in the brain, so excess needs to be removed. Filtration at the level of capillaries via the blood-brain barrier also allows fluid to enter the brain interstitial space, a process that might subtly increase in many disease states, including Alzheimer disease and small vessel disease. Fluid can enter the interstitial space directly from the ventricles, as in ventricular obstruction, although this route is unlikely to be a major contributor to physiological interstitial fluid. Fluid also enters the brain from CSF via the perivascular spaces as part of the glymphatic flushing system, as explained in our Review ${ }^{1}$. Therefore, it is possible that some fluid has passed through several intracranial compartments before exiting via one of the several exit routes (the arachnoid granulations to venous sinuses, the meningeal lymphatics along the dural sinuses, or the nasal lymphatics ${ }^{5}$ ), which compounds the difficulties of measuring the source of fluid.

Measurement of intracranial fluid production is difficult. In humans, opening of the cranial cavity, even with small burr holes, disturbs the usual pressure balance between intravascular and extravascular compartments ${ }^{8}$, so might also perturb blood and fluid transit through the various compartments. Cranial windows in rodents affect brain temperature, thus altering blood flow and cellular activity ${ }^{9}$, so might also affect fluid production and movement. The temperature effects persist after re-closure of the cranial cavity with a glass window that is sealed with dental cement or by substituting the window by thinning the cranium ${ }^{9}$, and these processes could also affect pressures, as the properties of these materials differ from those of the intact skull. Improvements in non-invasive medical imaging techniques, such as recent non-invasive approaches to the measurement of fluid crossing the blood-brain barrier and blood-CSF barrier ${ }^{10}$, the use of multi-pronged approaches, and awareness of the limitations of each approach, will clarify the importance of each intracranial fluid production and drainage route in the near future.

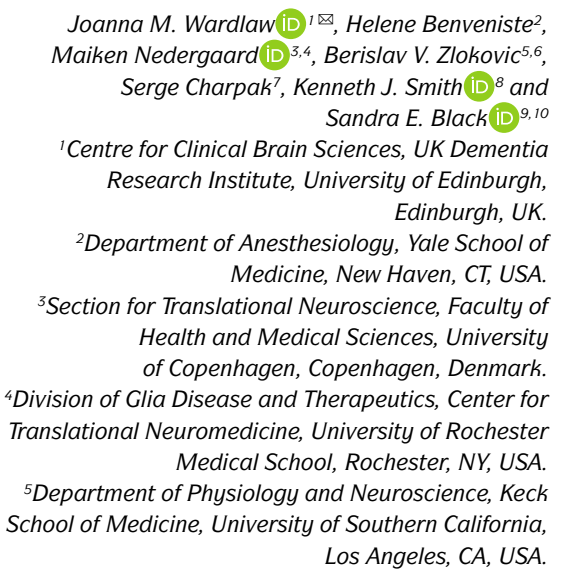

${ }^{6}$ Zilkha Neurogenetic Institute, Keck School of Medicine, University of Southern California, Los Angeles, CA, USA. 'INSERM, CNRS, Institute de la Vision, Paris, France. ${ }^{8}$ Department of Neuroinflammation, UCL Queen Square Institute of Neurology, London, UK. ${ }^{9}$ Department of Medicine (Neurology), Sunnybrook Health Sciences Centre, University of Toronto, Toronto, ON, Canada.

${ }^{10}$ Centre for Brain Resilience and Recovery, Hurvitz Brain Sciences Research Program, Sunnybrook Research Institute, University of Toronto, Toronto, ON, Canada. ${ }_{\text {e-mail: Joanna.Wardlaw@ed.ac.uk }}$ https://doi.org/10.1038/s41582-021-00539-z

1. Wardlaw, J. M. et al. Perivascular spaces in the brain: anatomy, physiology and pathology. Nat. Rev. Neurol. 16, 137-153 (2020)

2. Roques, M., De Barros, A. \& Bonneville, F. Rethink the classical view of cerebrospinal fluid production. Nat. Rev. Neurol. https://doi.org/10.1038/s41582021-00538-0 (2021)

3. Fox, R. J., Walji, A. H., Mielke, B., Petruk, K. C. \& Aronyk, K. E. Anatomic details of intradural channels in the parasagittal dura: A possible pathway for flow of cerebrospinal fluid. Neurosurgery 39, 84-91 (1996).

4. Rasmussen, M. K., Mestre, H. \& Nedergaard, M. Fluid transport in the brain. Physiol. Rev. https://doi.org/10.1152/physrev.00031.2020 (2021).

5. Ahn, J. H. et al. Meningeal lymphatic vessels at the skull base drain cerebrospinal fluid. Nature $\mathbf{5 7 2}$, 62-66 (2019).

6. Liu, G. et al. Direct measurement of cerebrospinal fluid production in mice. Cell Rep. 33, 108524 (2020).

7. Bradbury, M. W., Cserr, H. F. \& Westrop, R. J. Drainage of cerebral interstitial fluid into deep cervical lymph of the rabbit. Am. J. Physiol. 240, F329-336 (1981).

8. Wardlaw, J. M., Cannon, J., Statham, P. F. X. \& Price, R. Does the size of intracranial aneurysms change with intracranial pressure? Observations based on colour "power" transcranial Doppler ultrasound. J. Neurosurg. 88, 846-850 (1998).

9. Roche, M. et al. In vivo imaging with a water immersion objective affects brain temperature, blood flow and oxygenation. Elife 8, e47324 (2019).

10. Evans, P. G. et al. Non-invasive MRI of bloodcerebrospinal fluid barrier function. Nat. Commun. 11, 2081 (2020).

\section{Acknowledgements}

The authors acknowledge the Fondation Leducq Transatlantic Network of Excellence for the Study of Perivascular Spaces in Small Vessel Disease (grant reference 16 CVD 05).

\section{Competing interests}

The authors declare support from academic grants but have no other competing interests. 